\title{
Entwurf eines neuen Bundesdatenschutzgesetzes
}

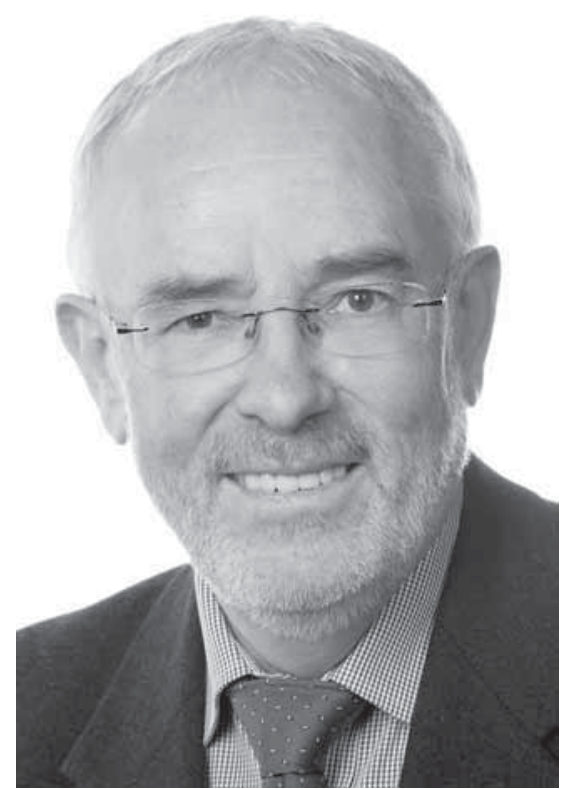

Viele wollen es nicht wahrhaben, weil es ihren Hoffnungen nicht entspricht. Aber die Verordnung (EU) 2016/679 des Europäischen Parlaments und des Rates vom 27. April 2016 zum Schutz natürlicher Personen bei der Verarbeitung personenbezogener Daten, zum freien Datenverkehr und zur Aufhebung der Richtlinie 95/46/EG (Datenschutz-Grundverordnung) bietet kein unionsweit einheitliches und kohärentes Datenschutzrecht und auch keinen Neuanfang für einen zukunftsfähigen Datenschutz. In ihrer verabschiedeten Form bietet sie nur einen Rahmen für eine Ko-Regulierung des Datenschutzes durch die Union und die Mitgliedstaaten. Als Grundverordnung regelt sie den Datenschutz nur im Grundsatz abschließend und ist in sehr vielen Details ergänzungsbedürftig. Mit 70 Öffnungsklauseln für die Mitgliedstaaten und vielen unbestimmten und ausfüllungsbedürftigen Rechtsbegriffen ist sie eine Richtlinie im Gewand einer Verordnung.

Die Datenschutz-Grundverordnung ist für ihre Umsetzung in Wirtschaft und Verwaltung, für ihre Vollzugsfähigkeit in der Praxis und für die Realisierung ihrer wenigen Reformansätze auf unterstützende nationalstaatliche Regelung angewiesen. Nur wenn die Mitgliedstaaten die neuen Steuerungsansätze wie die datenschutzgerechten Gestaltungen von Technik und Organisation der Datenverarbeitung und die datenschutzfreundlichen Voreinstellungen von IT-Systemen, die DatenschutzFolgenabschätzung und die Zertifizierung von Datenverarbeitungsvorgängen konstruktiv und reformorientiert aufgreifen und ihre Regelungsspielräume aktiv für eine Modernisierung des Datenschutzrechts nutzen, werden diese Wirklichkeit werden.

Für die Mitgliedstaaten in der Union hat der Datenschutz in der Bundesrepublik Deutschland Vorbildfunktion. Dies wird für die Umsetzungsgesetze zur DatenschutzGrundverordnung erst recht gelten. Kleinere Mitgliedstaaten, die im Datenschutz nicht so weit entwickelt sind, werden sich an dem Umsetzungsgesetz in Deutschland orientieren. Ob die reformorientierten Ansätze der Verordnung in der Europäischen Union in der Praxis realisiert werden, hängt stark von den einschlägigen legislativen Maßnahmen in Deutschland ab. Die Umsetzung des Regelungsimpulses der Verordnung in ein neues Bundesdatenschutzgesetz wird auch als Vorbild für die Datenschutzregelungen in den Ländern und für die Überarbeitungen der bereichsspezifischen Datenschutzgesetze wirken.

Dem Entwurf eines neuen Bundesdatenschutzgesetzes, den die Bundesregierung am 1. Februar 2017 in das Gesetzgebungsverfahren eingebracht hat, kommt daher eine große rechtspolitische Bedeutung zu. Er ist als Art. 1 eingebettet in den Entwurf eines Artikelgesetzes zur Anpassung des Datenschutzrechts an die Verordnung (EU) 2016/679 und zur Umsetzung der Richtlinie (EU) 2016/680 (DatenschutzAnpassungs- und -Umsetzungsgesetz EU - DSAnpUG-EU). Der Entwurf eines neuen Bundesdatenschutzgesetzes enthält in Teil 1 gemeinsame Bestimmungen, in Teil 2 Anpassungen des allgemeine Datenschutzrechts des Bundes an die DatenschutzGrundverordnung und in Teil 3 die Umsetzung der Richtlinie (EU) 2016/680 zum Datenschutz im Bereich der Justiz und Polizei im allgemeinen Datenschutzrecht des Bundes. Er ist mit 85 Paragraphen beinahe doppelt so umfangreich wie das bisherige Bundesdatenschutzgesetz. Diese Neufassung soll das geltende Bundesdatenschutzgesetz mit Geltung der Datenschutz-Grundverordnung in den Mitgliedstaaten zum 28. Mai 2018 ablösen. Daher soll der vorgelegte Entwurf noch in dieser Legislaturperiode, wegen der Bundestagswahlen im September 2017 also vor der Sommerpause 2017, als Gesetz verabschiedet werden.

Da die Bundesregierung den Entwurf erst spät in das Gesetzgebungsverfahren eingebracht hat, bleiben für Bundestag und Bundesrat sowie für die Öffentlichkeit nur wenig Zeit, sich mit diesem so wichtigen Gesetzgebungsvorhaben intensiv auseinanderzusetzen und konstruktive Vorschläge zu entwickeln, die den Entwurf verbessern. Da das Gesetz alle Wirtschafts-, Verwaltungs- und Lebensbereiche betrifft, muss diese Diskussion gesellschaftsweit geführt werden und die Chance haben, vom 
Gesetzgeber noch berücksichtigt zu werden.

Zu dieser Diskussion wollen die Beiträge im Schwerpunkt dieses Heftes beitragen. Sie sind aus der Veranstaltung „Die Ergänzung der Datenschutzgrundverordnung in Deutschland - Was soll sich ändern und was bleiben?" hervorgegangen, die das Competence Center for Applied Security Technology (CAST) und das BMBFForum „Privatheit und selbstbestimmtes Leben in einer digitalen Welt" gemeinsam am 23. März 2017 in Darmstadt veranstaltet haben. Die Beiträge gehen auf Vorträge oder Diskussionsvoten zurück, die die Autoren für diesen Schwerpunkt ausgearbeitet haben. Sie analysieren und bewerten den Regierungsentwurf für ein neues Bundesdatenschutzgesetz vor der Fragestellung, welche Datenschutzregeln ab Mai 2018 für Wirtschaft und Verwaltung gelten sollen und wie diese in der Ko-Regulierung des Datenschutzrechts in der Union und in Deutschland erreicht werden können. Diese Erwartungen an das künftige Datenschutzrecht prägen die Bewertungen des Regierungsentwurfs zum Bundesdatenschutzgesetz. Untersucht wird, ob dessen Vorschläge den absehbaren Herausforderungen des Datenschutzes gerecht werden und ob sie für die Verantwortlichen, Betroffenen und Aufsichtsbehörden ausreichende Handlungsmöglichkeiten bieten. Vor allem steht im Mittelpunkt des Interesses, ob der deutsche Gesetzgeber die Regelungsmöglichkeiten der DatenschutzGrundverordnung ausreichend und sinnvoll ausschöpft oder ob und wie der Gesetzentwurf noch verbessert werden kann.

Der einleitende Beitrag von Roßnagel stellt die Aufgaben und Spielräume des deutschen Gesetzgebers zur Ergänzung der Datenschutz-Grundverordnung vor und untersucht den Entwurf danach, ob er die Aufgaben erfüllt und die Spielräume ausreichend nutzt. Weber geht als Datenschutzbeauftragter der Software AG der Frage nach, ob der Entwurf für ein neues Bundesdatenschutzgesetz den Interessen der Wirtschaft gerecht wird. Bieker und Hansen fragen nach der Leistungsfähigkeit des Entwurfs, für die künftigen technisch-organisatorischen Herausforderungen den richtigen Rahmen zu bilden, die neuen Instrumente wie die Datenschutz-Folgenabschätzung und die Zertifizierung richtig zu unterstützen und Anreize für Innovationen in der Datenschutztechnik zu setzen. Roßnagel untersucht die Forderung der Konferenz der unabhängigen Datenschutzbeauftragten, die Öffnungsklauseln der Verordnung dafür zu nutzen, die künftige Leistungsfähigkeit des Datenschutzes zu stärken. Er untersucht daher, ob die Öffnungsklauseln für öffentliche Interessen und für den Beschäftigungskontext genutzt werden könnten, um beispielhaft für die Union risikoadäquate Datenschutzregelungen zu erproben. Geminn prüft den Entwurf daraufhin, ob er in einem wichtigen Bereich der künftigen Datenverarbeitung - dem Internet der Dienste und Dinge - zukunftsweisende risikoadäquate Lösungen bietet. Einen bestimmten Anwendungsbereich haben sich auch Johannes und Richter ausgewählt. Sie setzten bei den besonderen Verarbeitungssituationen an, deren Regulierung die Datenschutz-Grundverordnung weitgehend den Mitgliedstaaten überlässt, und analysieren, ob der Entwurf für Forschung, Statistik und Archivierung adäquate Regelungen enthält.

Alle Beiträge kommen - auf unterschiedlichen Wegen und mit unterschiedlicher Gewichtung - letztlich zu dem Ergebnis, dass der Entwurf für ein neues Bundesdatenschutzgesetz den hohen Erwartungen an eine Reform des Datenschutzrechts in der Union und in Deutschland nicht gerecht wird. Sie wenden diese Kritik konstruktiv und entwickeln Vorschläge, wie der Entwurf noch im Gesetzgebungsverfahren verbessert werden kann und wie die Datenschutzdiskussion in der neuen Legislaturperiode nach den Bundestagswahlen fortgeführt werden muss.

\section{Alexander Roßnagel}

